\title{
Testes de Atenção para Idosos: Relações com Idade, Escolaridade e Moradia
}

\author{
Eliane Sousa de Oliveira Fernandes \\ Acácia Aparecida Angeli Santos \\ Universidade São Francisco \\ Bragança Paulista, SP, Brasil
}

\begin{abstract}
RESUMO
O estudo se propôs a buscar evidências de validade para testes de atenção concentrada e sustentada em idosos, pelos critérios idade, escolaridade, condições de moradia (abrigados ou não) e tempo de resposta do instrumento, assim como validade convergente pela correlação entre eles. Participaram 83 pessoas de 60 anos ou mais, ambos os sexos. Foram aplicados o teste de Atenção Concentrada (AC) e o Teste de Atenção Sustentada (TASU). Os escores de ambos se correlacionaram negativamente com idade, e positivamente com escolaridade. Os idosos jovens tiveram melhor performance que os longevos e os não abrigados apresentaram maiores médias. No AC, com $50 \%$ a mais de tempo para os longevos, o desempenho não diferiu significativamente dos mais jovens. A correlação entre AC e TASU indicou que as medidas são convergentes. Os resultados obtidos permitiram concluir que ambas as medidas foram suficientemente sensíveis para avaliação da atenção em idosos.
\end{abstract}

Palavras-chave: Atenção concentrada; Atenção sustentada; Testes cognitivos; Envelhecimento.

\begin{abstract}
Attention Tests for Elderly People: Relationships with Age, Education and Housing

The purpose of the study was to find validity evidence for tests measuring focused and sustained attention in the elderly, based on the criteria age, education, housing conditions (sheltered or not), and response time on the instrument, as well as convergent validity through the correlation among them. Eighty-three people participated in the study, over 60 years of age and of both genders. The tests applied were the Focused Attention (FA) test and the Sustained Attention Test (SA). Scores on both correlated negatively with age and positively with education. The younger participants performed better than the older participants, and the homeless had higher averages. On the FA test, with a $50 \%$ longer response time for the older participants, the performance did not differ significantly from the younger. The correlation between the FA and the SA indicated that both measures are convergent. The results permit the conclusion that both measures are sensitive enough to assess attention in the elderly.
\end{abstract}

Keywords: Focused attention; Sustained attention; Cognitive tests; Aging.

\section{RESUMEN}

\section{Pruebas de Atención para Ancianos: Relaciones com la Edad, la Escolaridad y la Vivienda}

El estudio buscó evidencia de validez para testes de atención concentrada y sostenida en ancianos, por los criterios edad, escolaridad, condiciones de morada (albergados o no) y tiempo de respuesta al instrumento; y validez convergente por la correlación entre los testes. Participaron 83 personas de 60 años o más, de ambos sexos. Se aplicó una prueba de Atención Concentrada (AC) y una de Atención Sostenida (TASU). Los testes se correlacionaron negativamente con la edad y positivamente con la escolaridad. Los ancianos más jóvenes tuvieron un desempeño mejor en los dos testes, y los que no estaban albergados presentaron mayores promedios. En el AC con 50\% a más para los longevos, y el desempeño no presentó diferencias significativas de los más jóvenes. La correlación entre AC y TASU indicó que las dos medidas son convergentes. Los resultados mostraron que ambas medidas eran sensibles para evaluar la atención en los ancianos.

Palabras clave: Atención concentrada; Atención sostenida; Pruebas cognitivas; Envejecimiento. 
A atenção tem sido objeto de interesse da Psicologia desde seus primórdios, havendo referência sobre seu estudo já no século XIX por autores como William James (1890). Ela é tida como imprescindível para a interação eficiente do indivíduo com o seu ambiente e considerada como um componente central do funcionamento cognitivo, da integração das funções mentais, assim como pré-requisito importante para a manifestação das habilidades intelectuais (Mesulam, 2000). Do ponto de vista dos processos psicológicos, a atenção se relaciona à consciência, afeto, motivação, memória, percepção, dentre outros (Gazzaniga \& Heatherton, 2005; Mesulam, 2000; Sternberg, 2008), consistindo em um processo multideterminado, responsável pela administração da grande quantidade de estímulos aos quais é exposto o organismo, tanto do meio externo, por meio dos órgãos do sentido, quanto do meio interno, como informações da memória e de outros processos cognitivos (Mesulam, 2000; Sternberg, 2008; Strauss, Sherman, \& Spreen, 2006). $\mathrm{Na}$ maioria dos modelos, a atenção se refere a um complexo sistema de processamento, que permite ao indivíduo filtrar informações relevantes e irrelevantes para direcionar ações internas e intenções, realizar e manipular representações mentais, e monitorar ou modular respostas aos estímulos (Gazzaniga \& Heatherton, 2005; Lezak, Howieson, \& Loring, 2004; Strauss et al., 2006).

A atenção é comumente utilizada para se referir à seletividade do processamento da informação, sendo este o sentido já enfatizado por James (1890), referindo que o processo de atenção se relaciona à seleção das informações mais relevantes do ambiente para lidar de forma mais eficiente com este. Segundo Sternberg (2008), essa característica estaria relacionada ao controle voluntário da atenção, envolvendo um processamento intencional dos estímulos mais importantes em determinado momento e contexto. Tão significativo quanto a seletividade, seria a capacidade que um indivíduo tem de selecionar um estímulo diante de muitos outros e conseguir manter-se focado nele por um intervalo de tempo. De acordo com Cambraia (2003), essa capacidade caracterizaria uma modalidade atencional, a atenção concentrada. No entanto, Braga (2007) salienta que quando essa capacidade envolver a manutenção do foco atencional por um longo período de tempo caracterizaria outra modalidade, a atenção sustentada.

Tem sido essencial o papel da neurobiologia para a compreensão dos processos cognitivos, dentre eles, a atenção. Como as demais funções cognitivas, há etapas de aquisições, baseadas no amadurecimento cerebral, assim como de declínio e perdas com o processo de envelhecimento. Como a maioria dos processos cognitivos, a capacidade atencional também se modifica com o envelhecimento (Argimon, Irigaray, \& Stein, 2014; Commodari \& Guarnera, 2008; Fonseca, Zimmermann, Scherer, Parente, \& Ska, 2010; Noronha, Sisto, Bartholomeu, Lamounier, \& Rueda, 2006; Rueda \& Castro, 2010; Sisto, Castro, Cecílio-Fernandes, \& Silveira, 2010). Com relação ao desenvolvimento cognitivo ao longo do ciclo da vida, existe um acréscimo, em termos de desempenho funcional, até a fase da adultez jovem, que corresponde à faixa entre 20 e 40 anos de idade (Papalia \& Olds, 2000). Após essa fase, começam a ocorrer perdas decorrentes do processo de envelhecimento (Camargo, Gil, \& Moreno, 2006; McGaughy \& Eichenbaum, 2002). Mostra-se relevante, portanto, na avaliação da atenção, uma compreensão de seus aspectos desenvolvimentais, especialmente para aqueles que lidam com os idosos, devendo se considerar a trajetória de desenvolvimento das habilidades atencionais nas faixas etárias mais avançadas (Strauss et al., 2006).

O envelhecimento normal é diferenciado pela variabilidade das funções cognitivas entre os indivíduos. A educação, o nível de atividade e os fatores genéticos, dentre outros aspectos, são variáveis que, de um modo ou de outro, influenciam as distintas capacidades ao longo da vida e determinam o estado na velhice. O envolvimento com exercícios físicos, atividades culturais e religiosas e a manutenção de contato com familiares e amigos parece contribuir como um fator de proteção de suas condições cognitivas (Bartrés-Faz, Clemente, \& Junqué, 1999; Souza, Borges, Vitória, \& Chiappetta, 2009). Com o objetivo de analisar as modificações no perfil de algumas habilidades cognitivas (percepção, fluência verbal, memória e atenção) em indivíduos muito idosos (80 anos ou mais), em um período de três anos, Argimon e Stein (2005) realizaram um estudo com delineamento longitudinal, que examinou os idosos antes e após o período citado, utilizando os mesmos instrumentos. Os resultados permitiram a confirmação da hipótese de que características sociodemográficas do grupo, como o envolvimento com a comunidade, atividades de lazer, convívio com familiares e atividades físicas, atuam como fatores de proteção às perdas cognitivas.

Outro aspecto que contribui de forma significativa como um fator de proteção para esse declínio é a escolaridade. Os indivíduos que tinham mais anos de escolarização conservaram um melhor resultado no período de três anos em muitas das funções cognitivas examinadas, sendo isso especificamente observado em relação à atenção. Os achados confirmaram resultados de estudo anterior de Argimon (2002). 
Também focalizando a escolaridade, Mello, Haddad e Dellaroza (2012) encontraram resultados convergentes. Os autores verificaram o estado cognitivo dos idosos por meio do Mini-Exame do Estado Mental (MEEM), associado a uma escala de Atividades Básicas de Vida Diária (AVD) de Katz. Melhor estado cognitivo e menor dependência nas atividades de vida diária foram identificadas nas pessoas com maior nível de escolaridade. $\mathrm{O}$ desempenho no teste cognitivo em analfabetos e alfabetizados mostrou uma influência significativa da escolaridade, com um aumento de quase $80 \%$ na performance no MEEM.

Considerando outro aspecto sociodemográfico referente a condições de moradia dos idosos, o estudo de Reis, Torres, Araújo, Reis e Novaes (2009) avaliou o estado mental de idosos institucionalizados, relacionando o fator escolaridade com o desempenho cognitivo. A amostra foi constituída por 60 idosos compreendidos na faixa etária de 60 a 69 anos (41,7\%), 70 a 79 anos (33,3\%), 80 a 89 anos $(11,7 \%)$ e acima de 90 anos (13,3\%). Os resultados mostraram que a maioria dos idosos institucionalizados $(60 \%)$ apresentaram algum tipo de comprometimento cognitivo. No que diz respeito à escolaridade, $45 \%$ dos idosos com alteração cognitiva, não sugestiva de déficit, eram analfabetos.

Considera-se que a população idosa se concentra em uma faixa etária de aproximadamente 30 anos, sendo necessária a subdivisão entre idosos jovens (60 a 79 anos) e os mais idosos ou longevos ( 80 anos ou mais) (Camarano, 2002; Ferrari, 2002). Os idosos mais velhos estão no limite da sua capacidade funcional, sendo o envelhecimento longevo marcado por uma aceleração no declínio funcional e cognitivo (Baltes \& Smith, 2003; Karlamangla et al., 2009). Torna-se necessário, portanto, em estudos com idosos, como o aqui realizado, verificar as diferenças entre os subgrupos idosos jovens e longevos, principalmente na avaliação de aspectos cognitivos. Além da influência da idade, da escolaridade e situações de institucionalização no desenvolvimento cognitivo, como visto até o momento, autores como Quevedo, Martins e Izquierdo (2006) alertam que alterações decorrentes do declínio motor e sensorial, comumente observadas na população de idosos, podem causar vieses em testes empregados para avaliar funções cognitivas nessa faixa etária.

Declínios tanto cognitivo como motor, relacionados à idade e à escolaridade, foram constatados no estudo de Rodrigues, Ferreira e Haase (2008) que investigaram adultos neurologicamente saudáveis, de 18 a 90 anos e com escolaridade entre um e 25 anos. Os resultados dos testes cognitivos e motores indicaram que quanto maior a idade, menor o desempenho. Também houve correlação positiva entre escolaridade e os testes que avaliam tanto a função cognitiva como motora, indicando que altos níveis de instrução correspondem a melhores resultados nos testes. As diferenças educacionais, contudo, foram mais robustas para a explicação do desempenho cognitivo ao passo que as diferenças etárias foram mais importantes para o desempenho motor.

A necessidade de compreender mais amplamente as questões atinentes ao desempenho cognitivo dos idosos, dado o aumento da população idosa mundial e nacional, justifica a relevância de se estabelecer evidências de validade para medidas de processos cognitivos para essa população. Vale lembrar que os dados do Instituto Brasileiro de Geografia e Estatística - IBGE (2011a) revelam que o percentual de brasileiros com mais de 60 anos de idade cresceu de $8,6 \%$ no ano 2000 para $11 \%$ em 2010, que equivale à aproximadamente 20 milhões de pessoas. Até o ano de 2020 o crescimento estimado é de $40 \%$, podendo chegar a 28 milhões de idosos. Além disso, o aumento da população muito idosa, com mais de 80 anos de idade (idosos longevos), estimada para 2020 é de 4 milhões de longevos, um aumento em torno de 38\% (IBGE, 2011b).

Torna-se, então, imprescindível olhar para essa população, investigando as patologias, mas, também, o desenvolvimento típico nessa faixa etária, buscando não só a prevenção de problemas de saúde mas, principalmente, promovendo condições para uma vida ativa e com qualidade. Com base nessas considerações, constata-se a importância de se avaliar adequadamente a atenção, especialmente em indivíduos idosos, visto que falar de qualidade de vida na velhice engloba a necessidade de estabelecer critérios para diferenciar o que é esperado do que pode ser classificado como excepcional nessa faixa etária, considerando-se a noção de envelhecimento ótimo (Neri \& Yassuda, 2008). A investigação de processos cognitivos no idoso, incluindo a atenção, pode auxiliar nessa distinção, como por exemplo, na detecção precoce de quadros demenciais (Sánchez-Rodriguez \& Torrellas-Morales, 2011; Valls-Pedret, Molinuevo, \& Rami, 2010; Vázquez-Marrufo et al., 2011). Para tanto, alterações patológicas e modificações decorrentes do processo de envelhecimento normal precisam ser diferenciadas.

Testes de atenção construídos para avaliação de pessoas adultas são suficientemente sensíveis para discriminar a atenção em idosos? Sobre essa questão, o presente estudo tem por objetivo investigar evidências de validade de critério e convergente para testes de atenção em pessoas com 60 anos ou mais. Adotando o preconizado por Nunes e Primi (2010), para o primeiro caso foram definidas as variáveis externas, idade, escolaridade, condição de moradia e tempo de execução 
do teste, todas com estreita relação com a atenção, visando investigar se os instrumentos enfocados nesta pesquisa podem ser usados para fins diagnósticos ou preditivos. Quanto à validade convergente, que consiste na análise do grau de associação de um teste com outros que avaliam o mesmo construto ou construtos semelhantes, é esperado que estes testes apresentem altas correlações entre si, e exibam, portanto, coeficientes de magnitude forte, usualmente acima de 0,50. Avaliar os processos cognitivos dos idosos será possível apenas com instrumentos que tenham evidências de validade para essa população, pois apenas com tais evidências poderão fornecer interpretações confiáveis a partir de seus resultados (Urbina, 2007).

\section{MÉTODO}

\section{Participantes}

Participaram deste estudo 83 pessoas com idade de 60 anos ou mais, ambos os sexos, com condições motoras, visuais e auditivas que não impedissem de responder adequadamente aos testes e com capacidade de compreensão das instruções. O estudo foi desenvolvido com amostra não aleatória proveniente de duas instituições que abrigam idosos (15 participantes) e de dois grupos de idosos (68 sujeitos) de uma cidade do interior do estado de Minas Gerais. Foram considerados inelegíveis para a pesquisa, os idosos que, por conhecimento da equipe técnica da instituição e dos grupos de idosos, apresentassem comprometimento da motricidade e da visão que pudessem interferir na execução dos testes, assim como os portadores de déficits auditivos que dificultassem a comunicação. Também, foram excluídos os idosos apontados pela equipe como portadores de déficits cognitivos sugestivos de demência.

A média de idade dos abrigados foi de $78,13 \mathrm{e}$ desvio padrão de 10,96 (idade mínima de 63 anos e máxima de 100 anos), aproximadamente 10 anos a mais do que a dos idosos do outro grupo, com média de 68,91 anos e desvio padrão de 5,92 (idade variando de 60 a 87 anos). A média de anos de escolaridade dos não abrigados foi de 7,84 anos e desvio padrão de 4,7, sendo mais que o dobro da dos abrigados, cuja média foi de 2,93 anos e desvio padrão de 2,89. Dos 83 participantes, 10 não trabalhavam e não recebiam benefícios financeiros como aposentadoria ou pensão; os demais eram aposentados, pensionistas ou ambos. Dez dos participantes (12\% da amostra), além de receberem os benefícios, ainda trabalhavam. Dos idosos não abrigados, a maior parte, $47 \%$, morava com o companheiro, $16,9 \%$ moravam sozinhos e o restante com filhos ou outros parentes.

\section{Instrumentos}

1. Teste de Atenção Concentrada-AC (Rueda, 2013)

No teste de atenção concentrada (AC), a informação de atenção concentrada, fornecida por este teste, está relacionada à capacidade de um indivíduo em selecionar o que é relevante em meio a vários distratores, em um tempo pré-estabelecido. A medida de AC é obtida somando-se os estímulos alvos que foram assinalados e subtraindo os erros e omissões cometidos pela pessoa, no tempo de dois minutos. Nos estudos psicométricos da Bateria Psicológica para Avaliação da Atenção - BPA, participaram 1759 sujeitos, com idade variando de 6 a 82 anos, sendo a maior concentração $(51,4 \%)$ entre 14 e 30 anos. Com relação ao gênero, $52,8 \%$ eram do sexo feminino e $47,2 \%$, masculino. O quesito escolaridade abarcou desde o Ensino Fundamental incompleto até o Superior completo, incluindo, também, a Educação de Jovens e Adultos, sendo a maior frequência das pessoas com Ensino Superior completo ou incompleto $(43,1 \%)$. Foram feitos estudos de evidência de validade de critério com as variáveis idade e escolaridade, identificando que os níveis de atenção alteravam em função destas variáveis. Em relação à idade, verificouse um aumento no nível atencional (nos três tipos de atenção) até o início da vida adulta (por volta dos 25 anos), declinando após essa fase da vida. No que concerne à escolaridade, observou-se que aumento no nível de atenção correspondia a uma maior escolaridade.

\section{Teste de Atenção Sustentada - TASU} (Monteiro \& Santos, 2011)

O Teste de Atenção Sustentada, TASU (Monteiro \& Santos, 2011) é um teste de atenção que avalia a capacidade do indivíduo em manter o foco na mesma tarefa continuamente durante um período de tempo, sem deixar que a fadiga e estímulos distratores interfiram no seu desempenho final. O teste é composto por uma única tarefa, na qual o sujeito deverá identificar um determinado símbolo, previamente mostrado em um exemplo, em meio a vários outros que podem confundir ou distrair a atenção durante a atividade. Para tanto, são apresentados 10 blocos, cada um com 23 símbolos alvos e 77 distratores distribuídos aleatoriamente. Para cada bloco é dado um tempo de execução de 45 segundos. O importante desse procedimento é verificar se a pessoa mantém a mesma média de acertos na realização da tarefa em cada um dos blocos. Em relação às propriedades psicométricas, Monteiro (2012) realizou estudo para estabelecer evidência de validade baseada na estrutura interna do instrumento, com 314 motoristas de ônibus urbano e quatro 
trocadores, todos do sexo masculino e com idades variando entre 22 e 62 anos. Os resultados apontaram para uma diminuição do desempenho dos participantes no decorrer do teste, assinalando que o teste de fato mensura especificamente a atenção sustentada. A autora, também, fez estudo para verificar evidências de validade de critério, contando com 494 motoristas e quatro trocadores, do sexo masculino e de 22 a 66 anos de idade. Dentre as variáveis externas enfocadas, salienta-se o efeito da faixa etária, que revelou existir diferença estatisticamente significativa entre os grupos separados por faixa etária. Em relação a esse critério, os resultados apontaram que conforme o aumento da idade, menor o desempenho dos participantes no TASU, com participantes adultos.

\section{Procedimento de coleta dos dados}

O estudo teve início após autorização das instituições e aprovação do projeto pelo Comitê de Ética em Pesquisa da Universidade São Francisco, sob número 0156.0.142.000-11. A coleta de dados foi precedida pela obtenção do Termo de Consentimento Livre e Esclarecido. Para as pessoas que consentiram sua participação foi dada sequência na aplicação com o preenchimento do Questionário de identificação e de dados sociodemográficos. Os testes foram aplicados no mesmo dia, com intervalo de aproximadamente 15 minutos entre os dois para evitar interferência da fadiga. A aplicação foi feita de forma coletiva com os grupos de idosos, no horário que participavam das atividades do grupo e conforme acordado anteriormente com eles. Com os idosos abrigados, a aplicação teve que ser feita individualmente pela dificuldade em agrupá-los num mesmo horário, devido à dinâmica das instituições.

A aplicação do AC foi feita em três fases. A primeira com o tempo definido pelo manual do teste, parando a marcação dos estímulos aos dois minutos. $\mathrm{O}$ cronômetro era parado e era solicitado aos testandos que escrevessem o algarismo " 2 " acima do símbolo onde fizeram a última marcação. Em seguida, davase continuidade à marcação do ponto em que haviam parado e a cronometragem do tempo, parando esta aos três minutos e escrevendo o algarismo " 3 " acima do símbolo onde pararam desta vez. Seguia-se, então, a marcação e a cronometragem, interrompendo-se aos quatro minutos. O TASU foi aplicado em uma única fase; o teste conta com um tempo total de 7 minutos e 30 segundos, sendo que para cada bloco do teste foi dado um tempo de execução de 45 segundos.

\section{Procedimento de análise dos dados}

A análise dos dados foi feita por meio de estatística descritiva e inferencial. Para a busca de evidência de validade de critério, o desempenho nos testes foi relacionado às variáveis externas idade, escolaridade, condição de moradia e tempo de resposta. Foi feita a correlação da idade com a pontuação nos testes AC e TASU, utilizando a prova de correlação de Pearson. Recorreu-se à ANOVA para comparar o desempenho, nesses instrumentos, de grupos separados por nível de escolaridade. Recorreu-se à ANCOVA para analisar o desempenho nos dois testes em relação à moradia, controlando-se as variáveis idade e escolaridade. Também foi verificada validade convergente por meio da comparação com os resultados de outra medida do construto de atenção (AC e TASU). Para tanto, foram correlacionados os dados do $\mathrm{AC}$, instrumento de atenção concentrada, com os obtidos no TASU, de atenção sustentada pela prova de correlação de Pearson. $\mathrm{O}$ nível de significância de $p<0,05$ foi adotado para todas as provas usadas.

\section{RESULTADOS}

$\mathrm{Na}$ descrição dos resultados são apresentadas, inicialmente, análises referentes à evidência de validade de critério, para o Teste de Atenção Sustentada (TASU) e para o Teste de Atenção Concentrada (AC), pela análise das variáveis externas, sendo a primeira a idade e a segunda a escolaridade. Outra variável externa usada refere-se à condição de moradia (abrigado e não abrigado). Especificamente com relação ao AC foi verificado o tempo de resposta ao instrumento. Após, foi realizado o estudo de correlação entre os resultados dos instrumentos que avaliam os construtos de atenção concentrada (AC) e atenção sustentada (TASU), na busca de evidência de validade convergente entre ambos. Os resultados que se seguem são apresentados na ordem anunciada.

Com relação à idade dos participantes, na pontuação no TASU, em todas as cinco folhas, assim como na geral, o índice de correlação foi moderado e significativo e ficou entre $-0,33$ e $-0,38$, com nível de significância predominante de $p<0,001$. O teste AC, de dois e três minutos, mostrou uma correlação moderada e significativa, de $-0,41$ e $-0,42$ respectivamente. $O$ resultado mostrou que tanto o desempenho em atenção concentrada como em sustentada diminuiu com o aumento da idade.

Para verificar a relação entre os testes e a escolaridade dos participantes, os anos de escolarização foram agrupados em quatro categorias, sendo a primeira referente aos analfabetos ou com até um ano de escolaridade, seguindo os grupos de dois a oito anos, de nove a onze anos e de doze ou mais anos de escolarização. A maior frequência $(60,2 \%)$ foi de 
participantes com dois a oito anos de escolaridade, que incluiu quem tinha o Ensino Fundamental completo ou incompleto. A menor porcentagem $(8,4 \%)$ foi de analfabetos ou com até um ano de escolarização.

Recorrendo-se à prova de Tukey, identificou-se que a pontuação média obedeceu sempre à ordem hierárquica dos anos de escolaridade, subindo gradativamente da menor para a maior. Três grupos se diferenciaram significativamente, aqueles com até um ano de escolaridade, daquele com dois a oito anos e o de mais de doze anos de escolarização. Os escores médios não se diferenciaram do grupo de nove a onze anos com o anterior (dois a oito anos) e o posterior (doze ou mais anos). Essa diferenciação somente não ocorreu no teste AC com aplicação de quatro minutos, no qual houve separação apenas do grupo com até um ano de escolarização dos demais.

Como explicitado no objetivo, a condição de moradia também foi uma variável externa usada na comparação do desempenho nos testes de atenção (AC e TASU). Para tanto, os participantes foram divididos em dois grupos, um dos que residiam em instituição de abrigo ( $18,1 \%$ do total de sujeitos) e os que não se enquadravam nesta condição. Recorrendo à ANCOVA, obteve-se após o controle das variáveis idade e escolaridade um valor de $F(8,72)=2,238$, com $p=0,034, \eta^{2}=0,199$. O desempenho foi melhor no grupo não abrigado, apontando que os idosos não abrigados desta amostra apresentam melhores condições de atenção, tanto concentrada quanto sustentada, mesmo com o controle das variáveis idade e escolaridade.

Foi averiguado, relativo ao teste $\mathrm{AC}$ de atenção concentrada, o desempenho dos idosos jovens, de 79 anos ou menos de idade (71 sujeitos) e dos longevos, de 80 anos ou mais (12 pessoas), considerando-se a variável tempo de execução do teste. Para esse fim, foi utilizado o teste $t$ de Student, com nível de significância de 0,05 . Nos resultados do AC de dois minutos, os idosos jovens obtiveram $M=54,90( \pm 30,091)$ e os longevos $M=32,17$ ( $\pm 27,319)$, sendo $t=2,450 \mathrm{com}$ $p=0,016$. No AC de três minutos, para os idosos jovens, $M=80,11( \pm 39,683)$ e para os longevos $M=46,82$ $( \pm 40,233)$, com $t=2,682$ e $p=0,009$. Para o AC de quatro minutos, encontrou-se $M=88,07( \pm 43,509)$ para os idosos jovens e $M=57,00( \pm 44,774)$ para os longevos, $t=2,279$ com $p=0,025$. Verificou-se que o grupo de idosos jovens obteve escores mais elevados no AC nas três tomadas de tempo, indicando que este grupo apresenta melhores condições de atenção concentrada.

Observou-se, também, que a média dos longevos, com mais tempo de execução, vai se aproximando da média de execução aos dois minutos dos idosos jovens.
Recorreu-se, então, ao teste $t$ one sample, com nível de significância de 0,05 , para verificar o desempenho dos longevos quanto ao tempo de execução do instrumento. Para tanto, comparou-se o valor médio no AC de dois minutos dos idosos jovens com as pontuações no AC de dois, três e quatro minutos dos longevos. Nos resultados, houve diferença significativa entre o valor comparado e o grupo de longevos na execução do teste em dois minutos, com melhor desempenho do grupo mais jovem $(t=-2,883 \operatorname{com} p=0,015)$. Com três minutos de execução, a diferença já não se mostrou significativa $(t=-0,695$ com $p=0,502)$, assim como com quatro minutos $(t=0,162$ com $p=0,874)$, sendo que neste último caso, a pontuação dos mais idosos foi maior. Pode-se afirmar que com $50 \%$ a mais de tempo, o desempenho em atenção concentrada dos longevos, neste estudo, já não difere significativamente da performance dos idosos jovens.

Objetivou-se, ainda, a busca de evidência de validade convergente para os construtos de atenção concentrada e atenção sustentada por meio da correlação entre os escores dos testes AC e TASU. Os resultados dessa correlação estão demonstrados na Tabela 1.

\section{TABELA 1}

Correlação do Teste AC de atenção concentrada, com tempo de aplicação de dois, três e quatro minutos (AC2', AC3' e AC4'), com o Teste TASU de atenção sustentada $(\mathrm{N}=83)$

\begin{tabular}{ccccccc}
\hline \multirow{2}{*}{ Tempo } & \multicolumn{7}{c}{ TASU } \\
\cline { 2 - 7 } & folha 1 & folha 2 & folha 3 & folha 4 & folha 5 & total \\
\hline AC 2' & 0,69 & 0,71 & 0,71 & 0,75 & 0,70 & 0,75 \\
AC 3' & 0,68 & 0,69 & 0,69 & 0,73 & 0,71 & 0,74 \\
AC 4' & 0,65 & 0,63 & 0,65 & 0,67 & 0,69 & 0,70 \\
\hline
\end{tabular}

Nota: Todos os coeficientes de correlação de Pearson $(r)$ são significativos ao nível de $p<0,001$.

Os resultados evidenciaram índices de correlação positiva e forte entre os testes, podendo-se inferir que melhor performance em atenção concentrada corresponde a desempenho mais alto em atenção sustentada. Nas correlações entre o AC de dois, três e quatro minutos com todas as folhas do TASU, assim como com a pontuação geral deste, o índice de correlação ficou entre 0,63 e 0,75 , indicando uma correlação forte. Isso significa dizer que ambas as medidas são convergentes, como inicialmente se esperava.

\section{DISCUSSÃO}

Os resultados obtidos apontaram para diferenças significativas no desempenho em testes de atenção 
concentrada e sustentada com relação à variável idade, visto que o desempenho diminuiu com o avanço desta. Esses dados corroboram os encontrados no estudo de Noronha et al. (2006), que apontou para a influência da idade nos resultados de teste de atenção sustentada, embora não tenham encontrado esta associação para teste de atenção concentrada. Por sua vez, o estudo de Fonseca et al. (2008), ao analisar o desempenho de algumas funções cognitivas, entre elas a atenção concentrada, de adultos de 19 a 89 anos de idade, encontrou diferença significativa, com escores mais baixos a partir dos 60 anos.

A relação com idade também foi observada em estudos com outras modalidades de atenção, como no de Sisto et al. (2010), que visava analisar a relação entre atenção seletiva e idade, indicando que o aumento da idade corresponde a uma diminuição da capacidade desta modalidade atencional. E no de Rueda e Castro (2010), que envolveu a atenção alternada, e verificou que o aumento da idade correspondeu a uma diminuição da pontuação nesta modalidade de atenção. Além disso, nesse estudo, os autores evidenciarem a existência de um decréscimo atencional, independente do tipo de atenção avaliado.

Também foi verificado, no presente trabalho, que idosos longevos apresentaram desempenho mais baixo que os idosos jovens, tanto em atenção concentrada quanto em sustentada. Esses achados estão de acordo com os dados de Ferrari (2002), que cita que as pessoas com mais de 80 anos apresentam características fisiológicas, cognitivas, psicológicas e sociológicas que as diferenciam dentro do grupo de idosos. Também Baltes e Smith (2003) salientam que os idosos mais velhos estão no limite da sua capacidade funcional; e Karlamangla et al. (2009) que descrevem o envelhecimento após os 80 anos como uma continuidade do processo fisiológico marcada por uma aceleração na velocidade de perda da capacidade funcional e cognitiva. Da mesma forma, o estudo de Argimon et al. (2014) objetivou avaliar o desenvolvimento cognitivo de idosos em diferentes faixas de idade. Um dos processos cognitivos estudados foi a atenção e na comparação entre os grupos, as autoras encontraram que os idosos mais jovens, de 60 a 69 anos apresentaram escores significativamente mais altos que os longevos, com 80 anos ou mais.

Outro fator que se apresentou significativo foi a influência da escolaridade nos resultados dos testes de atenção. O desempenho dos participantes, tanto em atenção concentrada como em sustentada, foi mais elevado entre os idosos com mais escolaridade. Esses resultados estão em consonância com o estudo longitudinal de Argimon e Stein (2005), que evidenciou que os indivíduos que tinham mais anos de escolaridade conservaram um melhor resultado no período de três anos em muitas das funcões cognitivas examinadas, incluindo a atenção. Também são congruentes com os obtidos no estudo de Rodrigues et al. (2008) que objetivaram verificar o desempenho motor e o perfil cognitivo, incluindo medida de atenção sustentada, de adultos neurologicamente saudáveis, relacionados à idade e à escolaridade, e encontraram correlação positiva entre escolarização e testes que avaliavam a função motora e cognitiva. Os autores mostraram, que os testes cognitivos foram mais influenciados pela escolaridade do que pela idade e verificaram menor desempenho cognitivo em indivíduos com menos de 10 anos de escolarização.

Ainda em relação à escolaridade, os resultados aqui obtidos evidenciaram diferença significativa de desempenho entre três grupos, um com sujeitos com até um ano de escolaridade, o segundo com dois a oito anos e o terceiro com mais de doze anos de escolarização. Tais dados são coerentes com os do estudo de Argimon e Stein (2005) no qual as pessoas com até três anos de escolaridade obtiveram um resultado significativamente inferior quando comparadas com aquelas de quatro ou mais anos de escolarização. No mesmo sentido, Reis et al. (2009), ao avaliarem o estado mental de idosos institucionalizados, verificaram que $45 \%$ destes que apresentavam alteração cognitiva, embora não sugestiva de déficit, eram analfabetos. De forma similar, em estudo relativo à avaliação cognitiva de idosos institucionalizados, Mello et al. (2012) verificaram melhor estado cognitivo e menor dependência nas atividades de vida diária nas pessoas com maior nível de escolaridade, com diferença significativa no desempenho de analfabetos e alfabetizados, em favor destes últimos. Argimon et al. (2014), por sua vez, ao avaliarem o desenvolvimento cognitivo de idosos de 60 anos ou mais, encontraram que o nível educacional influenciou a performance nos testes cognitivos, inclusive o de atenção, apontando resultados mais baixos, com diferença significativa, no desempenho dos indivíduos com até três anos de educação formal.

Além da escolaridade, foi verificada no presente estudo, influência no desempenho dos testes em razão de outro fator sociodemográfico, a situação de moradia, se residente ou não em abrigos. Os resultados evidenciaram que os idosos não abrigados apresentam melhores condições de atenção tanto concentrada quanto sustentada, mesmo quando controladas as variáveis idade e escolaridade. Corroborando com esses achados, Reis et al. (2009) verificaram, no seu estudo, que $60 \%$ dos idosos institucionaliza 
dos apresentaram algum tipo de comprometimento cognitivo. A explicação para essa diferença pode estar na escassez, nessa população, de fatores considerados de proteção para o declínio cognitivo citado por autores como Argimon e Stein (2005), que salientam a importância do envolvimento com a comunidade, diferentes atividades de lazer, convívio com familiares e atividades físicas, raramente acessíveis aos idosos asilados. Independentemente da condição de moradia, Souza, Borges, Vitória e Chiappetta (2009) apontam para a importância de o idoso estar inserido em um ambiente onde ele realiza atividades de vida diária, exercícios físicos, participa de atividades culturais e religiosas e mantenha contato com familiares e amigos, pois são fatores de proteção de sua condição cognitiva.

Deve-se atentar, no entanto, para a heterogeneidade dos grupos de idosos abrigados e não abrigados, do atual estudo, em relação à idade e à escolaridade. Os indivíduos asilados tinham média de idade de aproximadamente dez anos a mais que o outro grupo e, quanto à escolaridade, por volta de cinco anos a menos de escolarização que os não asilados. Embora tenham sido encontrados resultados significativos no desempenho nos testes de atenção, mesmo quando controladas as variáveis idade e escolaridade, faz-se necessário estudo com grupos mais homogêneos para confirmar essa diferença.

$\mathrm{Na}$ avaliação cognitiva de idosos, deve-se considerar, como alertado por Quevedo et al. (2006), que ocorrem alterações motoras e sensoriais decorrentes do envelhecimento que podem causar vieses em testes que mensuram funções cognitivas nessa faixa etária. Levando em conta esse fato, foi que se propôs analisar o tempo de execução do instrumento de atenção concentrada (AC) pela comparação entre os dois grupos de idosos, os jovens e os longevos. Os resultados mostraram haver diferença significativa entre os dois grupos, com melhor desempenho do mais jovem, no tempo de execução estipulado pelo teste. No entanto, com $50 \%$ ou mais de tempo, o desempenho em atenção concentrada dos longevos já não diferiu significativamente da performance dos idosos jovens.

De acordo com Bottino, Cid e Camargo (1997), a partir da faixa etária de 70 anos, as reduções mais significativas nos indivíduos desta idade, se relacionam às habilidades práxicas e executivas que dependem de percepção visual, análise visuoespacial e desempenho visomotor, habilidades estas necessárias na execução do teste AC. Da mesma forma, Parusaraman e Greenwood (2000) relatam que a velhice traz alguma redução na velocidade de processamento e na flexibilidade. E de acordo com Camargo et al. (2006), essas reduções decorrem, também, de mudanças na atenção, na memória imediata e na capacidade de planejamento antecipatório das ações.

Corroborando com o resultado encontrado no presente trabalho encontra-se o estudo de Rodrigues, Ferreira e Haase (2008) que investigou o desempenho motor relacionado à idade e à escolaridade. Os resultados indicaram que quanto maior a idade, menor o desempenho cognitivo e maior o tempo de execução das tarefas, com declínio do desempenho motor em pessoas com mais de 60 anos de idade. Os autores também constataram que as diferenças educacionais foram mais robustas para a explicação do desempenho cognitivo ao passo que, para o desempenho motor, as diferenças etárias foram mais importantes.

Passando ao objetivo final deste estudo, qual seja, busca de evidências de validade convergente para os construtos de atenção concentrada e atenção sustentada, correlacionou-se os escores obtidos nos testes AC e TASU. De acordo com Nunes e Primi (2010), o objetivo principal de pesquisas de validade convergente é averiguar se um teste apresenta forte associação com outros instrumentos psicológicos que avaliam o mesmo construto. Se dois testes medem construtos idênticos, devem ter altas correlações entre si, os coeficientes devem ser de magnitude forte, acima de 0,50 . Os resultados do presente estudo evidenciaram correlações de todas as folhas do TASU, assim como da pontuação geral deste, com o AC de dois, três e quatro minutos, com índices de correlação entre 0,63 e 0,75 , o que significa um grau forte de correlação, indicando que ambas as medidas são convergentes. Os dados acima permitem inferir, também, que melhor performance em atenção concentrada corresponde a desempenho mais alto em atenção sustentada.

A evidência de validade convergente entre medidas de atenção sustentada e concentrada também foi evidenciada no estudo de Noronha et al. (2006) que correlacionou medidas de Atenção Sustentada - AS (Sisto et al., 2006) e de Atenção Concentrada - AC (Cambraia, 2003). Os resultados desse estudo mostrou que as medidas de concentração e a velocidade com qualidade do AS apresentaram correlações positivas e significativas, embora fracas ( $\mathrm{r}=0,28 \mathrm{e} \mathrm{r}=0,40$ respectivamente) com o $\mathrm{AC}$ de forma geral, com maior magnitude da correlação do AC com velocidade com qualidade.

Nos estudos psicométricos da Bateria Psicológica para Avaliação da Atenção - BPA (Rueda, 2013), na qual está inserido o teste de atenção concentrada utilizado neste trabalho, foi verificada a validade convergente por correlação dos testes que compõem a BPA com testes de atenção concentrada, dividida e alternada, evidenciando que todas as correlações foram significativas e de magnitude moderada. Os 
coeficientes de correlação entre os testes foram todos acima de 0,50. Do mesmo modo, foram feitos estudos de validade convergente para o Teste de Atenção Sustentada - TASU (Monteiro, 2012). Para tanto, foram utilizados instrumentos de medida de atenção sustentada, concentrada, alternada e dividida. Os resultados mostraram correlações de magnitude forte entre as folhas do TASU e os testes de atenção variando entre 0,52 e 0,67, configurando evidência de validade convergente, com exceção do teste de atenção dividida, cujo valor variou entre 0,20 e 0,37 , apontando um grau fraco de correlação.

Em síntese, pode se verificar que a diferença de faixa etária foi significativa na performance em testes de atenção concentrada e sustentada, com o desempenho diminuindo com o avanço da idade, sendo, também, relevante o desempenho mais baixo dos idosos longevos quando comparados aos idosos jovens. A escolaridade foi outro fator preponderante, visto que a performance nas duas modalidades atencionais foi mais elevada em idosos com mais anos de escolarização. Quanto à situação de moradia, foi evidenciado que os idosos não abrigados apresentavam melhores condições de atenção concentrada e sustentada, mas, também, apresentavam média de idade mais baixa e mais anos de escolaridade. Por fim, os resultados evidenciaram correlações positivas, significativas e de magnitude forte entre o teste de atenção sustentada, TASU, e o de atenção concentrada, AC, indicando que ambas as medidas são convergentes, como inicialmente se esperava.
Este estudo permitiu que se observasse o desempenho dos idosos em testes de atenção. Os resultados obtidos indicaram que há diferença de desempenho nas medidas de atenção concentrada e sustentada em relação à idade, mostrando que este diminui em função do envelhecimento. Deve-se apontar como limitação o número de sujeitos e o fato de serem todos de uma mesma cidade. Em relação aos asilados, foram poucos os idosos em condição de responder os testes, o que pode afetar a generalização dos achados.

Uma questão relevante para futuros trabalhos seria verificar as perdas cognitivas com o avanço da idade, especificando as diferenças entre as diversas funções, principalmente em relação às faixas de idade nas quais este declínio pode ser mais expressivo. Estudos de coorte, comparando indivíduos em idades diferentes podem ser enviesados por diferenças biológicas, cognitivas, socioculturais, como pela diferença entre as gerações. Estudos longitudinais, que avaliassem as mesmas pessoas em diferentes fases do desenvolvimento, incluindo a separação por idade, poderiam trazer maiores esclarecimentos sobre a questão levantada. A influência de fatores sociodemográficos aponta para a importância de se desenvolver estudos experimentais relacionandoos ao desempenho cognitivo. A literatura tem mostrado que alguns deles atuam como fatores de proteção para o declínio cognitivo, o que torna relevante que eles sejam mais bem investigados e que sejam desenvolvidos mais estudos focados em estratégias de intervenção, visando melhor qualidade de vida.

\section{REFERENCIAS}

Argimon, I. L. (2002). Desenvolvimento cognitivo na terceira idade. Tese de doutorado, Pontifícia Universidade Católica do Rio Grande do Sul, Porto alegre, RS, Brasil.

Argimon, I. L. \& Stein, L. M. (2005). Habilidades cognitivas em indivíduos muito idosos: um estudo longitudinal. Cadernos de Saúde Pública, 21(1), 64-72. http://dx.doi.org/10.1590/S0102-311X2005000100008

Argimon, I. L., Irigaray, T. Q., \& Stein, L. M. (2014). Cognitive development across different age ranges in late adulthood. Universitas Psychologica, 13(1), 253-264. http://dx.doi.org/10.11144/2338

Baltes, P. B. \& Smith, J. (2003). New frontiers in the future of aging: from successful aging of the young old to the dilemmas of the fourth age. Gerontology, 49, 123-125. http://dx.doi.org/10.1159/000067946

Bartrés-Faz, D., Clemente, I.., \& Junqué, C. (1999). Alteración cognitiva en el envejecimiento normal: nosología y estado actual. Revista de Neurologia, 29(1), 64-70.

Bottino, C. M., Cid, C. G., \& Camargo, C. H. (1997). Avaliação neuropsicológica. In O. V. Forlenza \& O. P. Almeida. Depressão e demência no idoso - Tratamento farmacológico e psicológico. São Paulo: Lemos Editorial.

Braga, J. L. (2007). Atenção Concentrada e Atenção Difusa: Elaboração de Instrumentos de Medida. Dissertação de Mestrado, Universidade de Brasília, Brasília, DF, Brasil.

Camarano, A. A. (2002). Envelhecimento da população brasileira: uma contribuição demográfica. Rio de Janeiro: Instituto de Pesquisa Econômica Aplicada (Textos para discussão, n. 858).

Camargo, C. H., Gil, G., \& Moreno, M. D. (2006). Envelhecimento "normal” e cognição. In C. M. Bottino, J. Laks, \& S. L. Blay, Demência e Transtornos Cognitivos no Idoso (pp. 13-20). Rio de Janeiro: Guanabara Koogan.

Cambraia, S. V. (2003). Coleção AC - Atenção Concentrada (3를. ed.). São Paulo: Vetor.

Commodari, E. \& Guarnera, M. (2008). Attention and aging. Aging clinical and Experimental Research, 6, 578-584. http:// dx.doi.org/10.1007/BF03324887

Fonseca, R. P., Zimmermann, L. C. S., Parente, M. A. M. P., \& Ska, B. (2010). Episodic memory, concentrated attention and processing speed in aging. Dement Neuropsychol, 4(2), 91-97. 
Gazzaniga, M. S. \& Heatherton, T. F. (2005). Ciência Psicológica. Mente, Cérebro e Comportamento. Porto Alegre: Artmed. IBGE. (2011b). Projeção da população. Acesso em 15 de agosto de 2011, disponível em Instituto Brasileiro de Geografia e Estatística: http://www.ibge.gov.br/home/estatística/população/projeção_da_população/2008/piramide/piramide.shtm

IBGE. (2011a). Sinopse dos Resultados do Censo 2010. Acesso em 15 de agosto de 2011, disponível em Instituto Brasileiro de Geografia e Estatística: http://www.censo2010.ibge.gov.br/sinopse/webservice

James, W. (1890). The Principles of Psychology. Acesso em 26 de setembro de 2010, disponível em Classics in The History of Psychology: http://psychclassics.yorku.ca/James/Principles/prin11.htm http://dx.doi.org/10.1037/11059-000

Karlamangla, A. S., Miller-Martinez, D., Aneshensel, C. S., Seeman, T. E., Wight, R. G., \& Chodosh, J. (2009). Trajectories of cognitive function in late in the US: demographic and socioeconomic predictors. American Journal of Epidemiology, 170(3), 331-342. http://dx.doi.org/10.1093/aje/kwp154

Lezak, M. D., Howieson, D. B., \& Loring, D. W. (2004). Neuropsychological Assessment (4th ed.). New York: Oxford.

McGaughy, J. \& Eichenbaum, H. (2002). It's Time to Pay Attention to Attention in Aging. Learning Memory, 9, 151-152. http:// dx.doi.org/10.1101/lm.52902

Mello, B. L., Haddad, M. C., \& Dellaroza, M. S. (2012). Avaliação cognitiva de idosos institucionalizados. Acta Scientiarum, 34(1), 95-102. http://dx.doi.org/10.4025/actascihealthsci.v34i1.7974

Mesulam, M.-M. (2000). Principles of behavioral and cognitive neurology (2nd ed.). New York: Oxford.

Monteiro, R. L. \& Santos, A. A. (2011). Teste de Atenção Sustentada, TASU. Relatório técnico da versão preliminar. Itatiba: Universidade São Francisco.

Neri, A. L. \& Yassuda, M. S. (2008). Velhice bem-sucedida: aspectos afetivos e cognitivos (3를. ed. Campinas: Papirus.

Noronha, A. P., Sisto, F. F., Bartholomeu, D., Lamounier, R., \& Rueda, F. J. (2006). Atenção sustentada e concentrada: construtos semelhantes? Psicologia: Pesquisa e Trânsito, 2(1), 29-36.

Nunes, C. H. \& Primi, R. (2010). Aspectos técnicos e conceituais da ficha de avaliação dos testes psicológicos. In A. A. Santos (Org.), Avaliação psicológica: diretrizes na regulamentação da profissão (pp. 101-128). Brasília: Conselho Federal de Psicologia.

Papalia, D. E. \& Olds, S. W. (2000). Desenvolvimento Humano (7ª ed.). São Paulo: Pioneira.

Parasuraman, R. \& Greenwood, P. M. (2000). Selective attention in aging and dementia. In R. Parasuraman. The Attentive Brain. Massachusetts: MIT Press.

Quevedo, J., Martins, M. R., \& Izquierdo, I. (2006). Alterações cerebrais e memória. In C. M. Bottino, J. Laks, \& S. L. Blay (Orgs.), Demência e transtornos cognitivos em idosos (pp. 3-12). Rio de Janeiro: Guanabara Koogan.

Reis, L. A., Torres, G. V., Araújo, C. C., Reis, L. A., \& Novaes, L. K. (2009). Rastreamento cognitivo de idosos institucionalizados no município de Jequié-BA. Psicologia em Estudo (Maringá), 14(2), 295-301. http://dx.doi.org/10.1590/S141373722009000200010

Rodrigues, J. L., Ferreira, F. O., \& Haase, V. G. (2008). Perfil do desempenho motor e cognitivo na idade adulta e velhice. Gerais: Revista Interinstitucional de Psicologia, 1(1), 20-33.

Rueda, F. J. \& Castro, N. R. (2010). Capacidade atencional: há decréscimo com o passar da idade? Psicologia Ciência e Profissão, 30(3), 572-587. http://dx.doi.org/10.1590/S1414-98932010000300010

Rueda, F. M. (2013). Bateria Psicológica para Avaliação da Atenção - BPA. São Paulo: Vetor.

Sánchez-Rodrigues, J. L. \& Torrellas-Morales, C. (2011). Revisión del constructo deterioro cognitivo leve: aspectos generales. Revista de Neurologia, 52(5), 300-305.

Sisto, F. F., Castro, N. R., Cecilio-Fernandes, D., \& Silveira, F. J. (2010). Atenção seletiva visual e o processo de envelhecimento. Cadernos de Pós-Graduação em Distúrbios do Desenvolvimento, 10(1), 93-102.

Souza, V. L., Borges, M. F., Vitória, C. M., \& Chiappetta, A. L. (2009). Perfil das habilidades cognitivas no envelhecimento normal. Revista CEFAC (São Paulo).

Sternberg, R. J. (2008). Psicologia Cognitiva. Porto Alegre: Artmed.

Strauss, E., Sherman, E. M., \& Spreen, O. (2006). A Compendium of Neuropsychological Tests (3rd ed.). New York: Oxford.

Urbina, S. (2007). Fundamentos da Testagem Psicológica. Porto Alegre: Artmed.

Valls-Pedret, C., Molinuevo, J. L., \& Rami, L. (2010). Diagnóstico precoz de la enfermidad de Alzheimer: fase prodrómica y preclínica. Revista de Neurologia, 51(8), 471-480.

Vázquez-Marrufo, M., Benítez, M. L., Rodríguez-Gómez, G., Galvao-Carmona, A., Fernández-Del-Olmo, A., \& VaqueroCasares, E. (2011). Afectación de las redes neurales atencionales durante el envejecimiento salutable. Revista de Neurologia, $52,20-6$.

Ward, A. (2004). Attention: A Neuropsychological Approach. New York: Psychology Press.

Autores:

ELIANE SOUSA DE OLIVEIRA FERNANDES - Doutoranda na Universidade São Francisco.

ACÁCIA APARECIDA ANGELI SANTOS - Doutora, Universidade São Francisco.

Endereço para correspondência:

Eliane Sousa de Oliveira Fernandes

Rua José Antônio de Barros, 70 - Pousada dos Campos

37550-000 Pouso Alegre, MG, Brasil

Recebido em: 13.05 .14

Aceito em: 13.10 .14

Psico, Porto Alegre, PUCRS, v. 46, n. 1, pp. 110-119, jan.-mar. 2015 